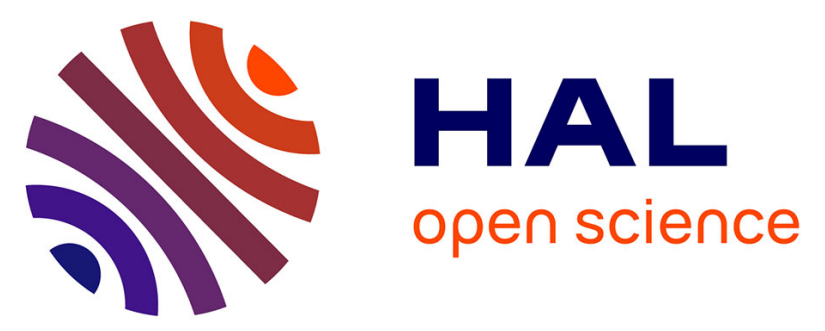

\title{
Novel circuit models of arbitrary-shape line: Application to parallel coupled microstrip filters with suppression of multi-harmonic responses
}

\author{
Marc Le Roy, André Pérennec
}

\section{- To cite this version:}

Marc Le Roy, André Pérennec. Novel circuit models of arbitrary-shape line: Application to parallel coupled microstrip filters with suppression of multi-harmonic responses. 35th European Microwave Conference, Oct 2005, Paris, France. pp.1-4, 10.1109/EUMC.2005.1610077 . hal-00809266

\section{HAL Id: hal-00809266 \\ https://hal.univ-brest.fr/hal-00809266}

Submitted on 8 Apr 2013

HAL is a multi-disciplinary open access archive for the deposit and dissemination of scientific research documents, whether they are published or not. The documents may come from teaching and research institutions in France or abroad, or from public or private research centers.
L'archive ouverte pluridisciplinaire HAL, est destinée au dépôt et à la diffusion de documents scientifiques de niveau recherche, publiés ou non, émanant des établissements d'enseignement et de recherche français ou étrangers, des laboratoires publics ou privés. 


\title{
Novel Circuit Models of Arbitrary-Shape Line: Application to Parallel Coupled Microstrip Filters with Suppression of Multi-Harmonic Responses
}

\author{
Marc Le Roy, André Pérennec \\ LEST (Laboratoire d'Electronique et Systèmes de Télécommunication) - UMR CNRS 6165. \\ UBO - ENSTBr, 6 Av. Le gorgeu, CS 93837, 29238 Brest Cedex 3, France. \\ E-mail: Marc.LeRoy@univ-brest.fr ; Phone: (33) 298016505 ; Fax: (33) 298016395.
}

\begin{abstract}
Following a hierarchical approach, several models are built in microwave-circuit design software to simulate and design many configurations of arbitrary-shape line. The global shape is controlled by a cubic spline interpolation; our purpose is, indeed, to make easier the handling of nonuniform structure for microwave device designers. To illustrate the potentialities of these new components, we designed a third-order bandpass coupledline filter at $5.5 \mathrm{GHz}$. A sinusoidal strip-width perturbation is applied by using the new model of nonuniform symmetrical coupled-line. Associated to over-coupling of the resonator end, the first three spurious passbands are then suppressed.
\end{abstract}

\section{INTRODUCTION}

Nonuniform transmission lines are mainly used as impedance-matching devices, pulse transformers, resonators, couplers and filters. Their great shape flexibility increases the number of degrees of freedom; on the other hand, it is somewhat difficult to manipulate due to the lack of accurate and general model. In a previous study [1], we proposed a specific model to get the S-parameters of a nonuniform line defined by a cubic spline interpolation. Lots of various curves and shapes can be precisely modeled with this method. But, this approach includes no losses, and the accuracy on dispersion effect modeling is insufficient. Moreover, a specific software is needed to generate the circuit layout. To overcome these problems while keeping the capacity of the cubic spline interpolation, we propose to build accurate models of arbitrary-shape lines for several technologies and configurations on the basis of existing software models. The great variety of basic models enables one to develop non-uniform models of microstrip or coplanar-waveguide single and coupled lines as well as multilayer arbitrary-shape structures. These new hierarchical components can be integrated in classical design and construction processes. Furthermore, the characteristic parameters of nonuniform lines can be easily controlled and optimized. The automatic layout generation allows the validation of circuit approach from electromagnetical simulations and avoids tedious and time-consuming hand-capture data. To validate the models and highlight their accuracy and easy handling, we designed a microstrip third-order coupled-line filter. Then, we applied a sinusoidal perturbation to the coupled lines to reject the spurious passbands. Compared to [2], the non-uniform coupled-lines remain symmetrical. Finally, the first three harmonic passbands were rejected by associating this perturbation technique with overcoupling of resonator end [3].

\section{FORMALISM AND HIERARCHICAL MODELS}

Our new models were developed under Advanced Design System (ADS) from Agilent; but, this approach can be transposed to any circuit software supporting hierarchical structures. This section will describe the major stages required to build a hierarchical model for a nonuniform microstrip line. The lowest level denoted Clevel is made of $m$ sections of microstrip tapers (fig. 1) for a substrate specified at the highest level. Dispersion, conductor loss, and dielectric loss effects are included in the microstrip taper model (linear width variation).

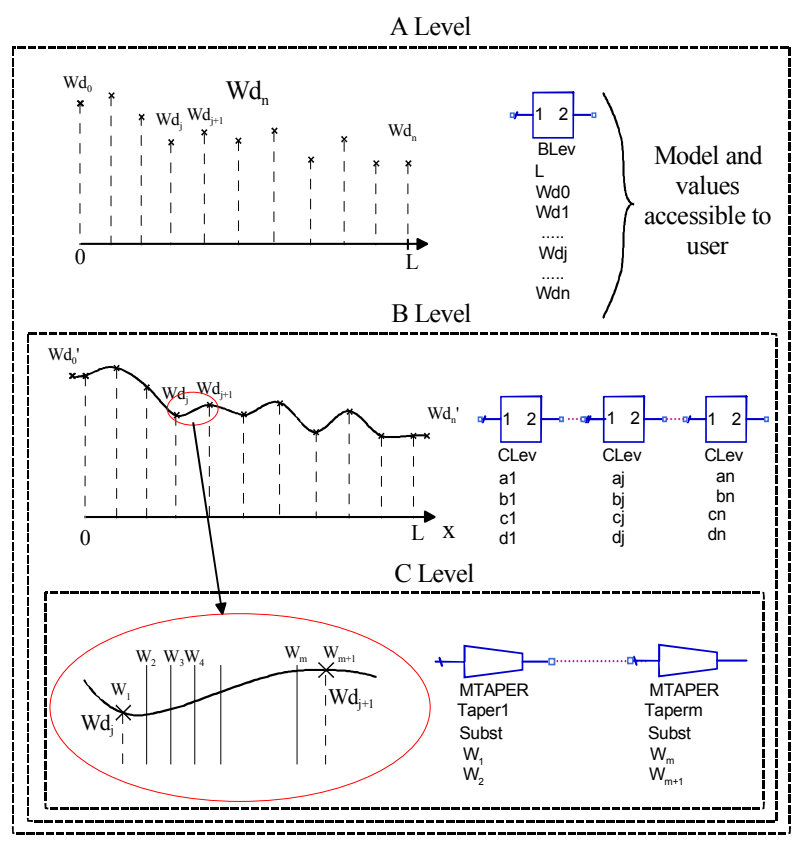

Fig. 1. Hierarchical structure of the model.

This C-level is saved as a black-box component termed CLev. The $m+1$ width values and the taper length are calculated at the $\mathrm{B}$ level which contains $n$ CLev 
components. At this stage, the cubic spline interpolation creates the line width curve that passes through a set of discrete values while minimizing the ripples. This interpolation also ensures the continuity of the first and second derivatives at these points. The $n+1$ discrete width values come from the A-level (fig. 1) and are defined by the user. Between 2 discrete points $W d_{j}$ and $W d_{j+1}$, the width variation is described by the following cubic polynomial:

$$
W(z)=a_{j}+b_{j} \cdot z+c_{j} z^{2}+d_{j} \cdot z^{3}
$$

From the $a_{j} \ldots d_{j}$ coefficients of the polynomial, the $m+1$ strip widths are extracted for the $j^{\text {th }}$ CLev component in B-Level. On fig. 1, it is worth noting the addition of virtual points $W d^{\prime}{ }_{0}$ and $W d^{\prime}{ }_{n}$ at the left- and right hand sides to create smooth transitions and connections to other components. In the example presented here, the total line length is split into cubic subsections of equal length; but, different length can be also considered to increase the number of degrees of freedom.

From the cubic spline interpolation and hierarchical structure described above, we developed many models (fig. 2) by changing only the built-in model (taper in fig. 1).

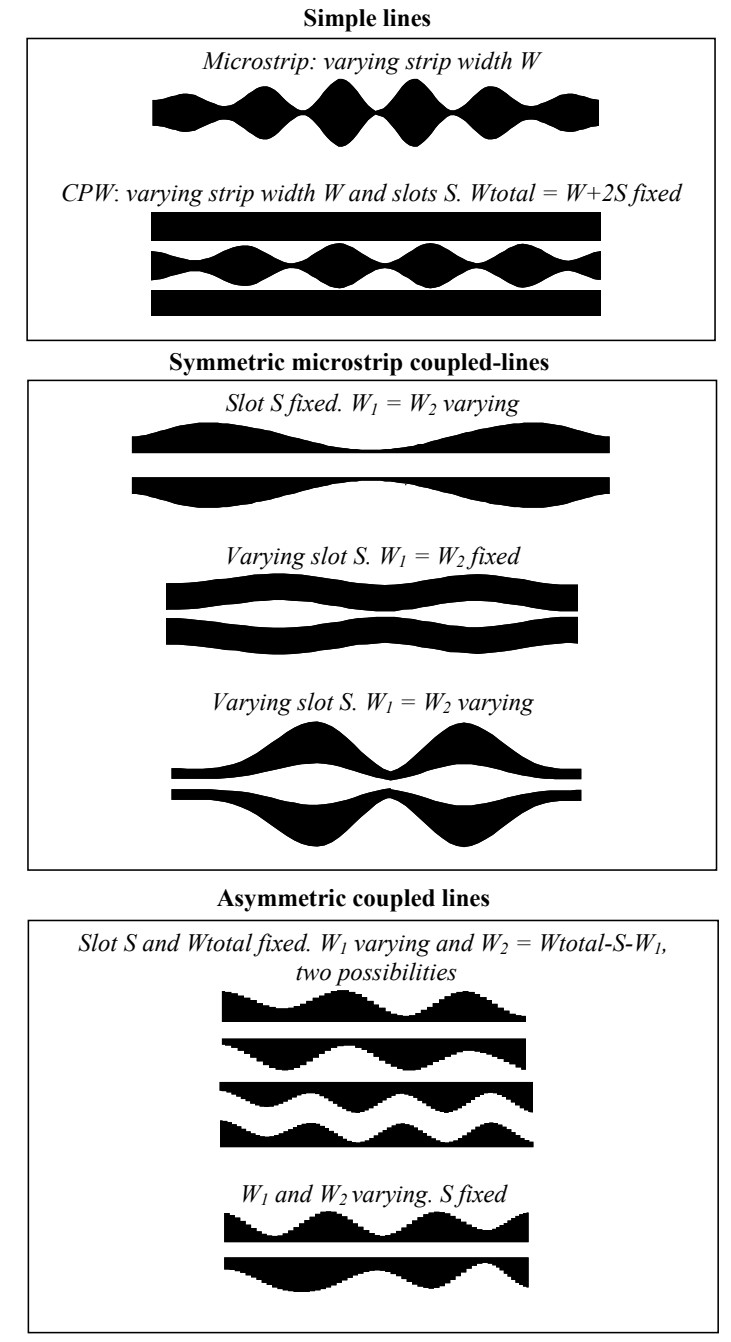

Fig. 2. Examples of new models.
The interpolation algorithm can be applied to strip width in microstrip or slots or strip in coplanar waveguide (CPW) and in coupled microstrip lines. Dual interpolation was also tested to control slots and strips of coupled-lines simultaneously. The number of possible combinations is too high to be depicted in fig. 2, particularly for CPW. However, we gathered them to create a new component library.

\section{APPLICATION TO PARALLEL COUPLED MICROSTRIP} FILTERS WITH MULTI-HARMONIC RESPONSES SUPPRESSION

To validate these hierarchical components and their integration in circuit software, a nonuniform coupled microstrip line component was implemented so as to improve the performances of a coupled-line bandpass filter. This third-order filter was designed for a Wi-Fi application with a bandwidth from 5.180 to $5.805 \mathrm{GHz}$ (11.4\% relative bandwidth) by following a classical design process. The main drawback of this kind of filter consists in spurious passbands at multiples of the passband central frequency $f_{c}$ due to a difference between the phase velocities of even- and odd-modes. For this specific topology, one of the most efficient techniques to suppress the first harmonic is to introduce overcoupled resonators as proposed in [3]; it increases the odd-mode length, but keeps constant the even-mode one. Figure 4 depicts the layout of this modified third-order Chebychev bandpass filter. Two overcoupled sections among the three available ones are sufficient to reject the first spurious response by more than $20 \mathrm{~dB}$ (at twice $f_{c}$ ) in simulation (dotted line in fig. 5). As specified in [3], the suppression of harmonics is more difficult when the permittivity of the microstrip filter substrate is high compared to a low one since the difference in phase velocities is ruled by $\varepsilon_{\mathrm{r}}$. Then, a sinusoidal variation around the nominal strip width value is introduced in order to suppress two other spurious harmonics. To reject the $k^{\text {th }}$ harmonics of $f_{c}$, the sinusoidal perturbation period $L$ is calculated as follows [2]:

$$
L=\frac{L_{R}}{k}
$$

where $L_{R}$ is the resonator length. Application of 1.5 sinusoidal periods to the two coupled-lines end-sections suppresses the $3 . f_{c}$ passband whereas that of 2 periods to the two central sections rejects the $4 . f_{c}$ harmonic. As done in [2], the strip width variation $w_{i}(z)$ is expressed in the $i^{\text {th }}$ coupled lines section and for a given modulation percentage $M_{i}(\%)$ :

$$
w_{i}(z)=w_{i}\left(1+\frac{M_{i}(\%)}{100} \cdot \cos \left(\frac{2 \pi z}{L_{i}}+\phi_{i}\right)\right)
$$

where $\phi_{i}$ is the initial phase, $w_{i}$ is the strip width of the conventional filter and $L_{i}$ is the period of the perturbation applied to the $i^{\text {th }}$ section. One should note that, here and contrarily to [2] the perturbation is applied to symmetrical sections; indeed, under this circuit software, the symmetrical coupled-lines model demonstrated a better accuracy than the asymmetric one. For an $N$-order 
filter, the sole use of the perturbation approach leads to the suppression of $N-1$ spurious passbands. Superposition of different periods on each resonator [4] doesn't allow going beyond and moreover, the spurious harmonics are less rejected than in [2]. Here, the combined use of overcoupled resonators and strip width modulation permits the rejection of three spurious responses for only an order-3 filter.

Figure 3 illustrates the filter schematic and shows the four hierarchical models of non-uniform symmetrical coupled-lines. The slot width is kept constant; the perturbation, thus the cubic spline interpolation, is applied to the strip widths for each coupled section.

\section{--..Order3Filter-A-Level (Schematic/Layout] \\ - -... B-Level (4 instances) \\ - .... C-Level (10 Instances)}

Fig. 3. Hierarchy of the three-order filter schematic.

These models allowed us to make a final optimization of the discrete point values (strip width) in order that the simulated circuit response fits at best the objective; in particularly to cancel the slight shift in frequency responses evidenced at the introduction of the sinusoidal perturbation.

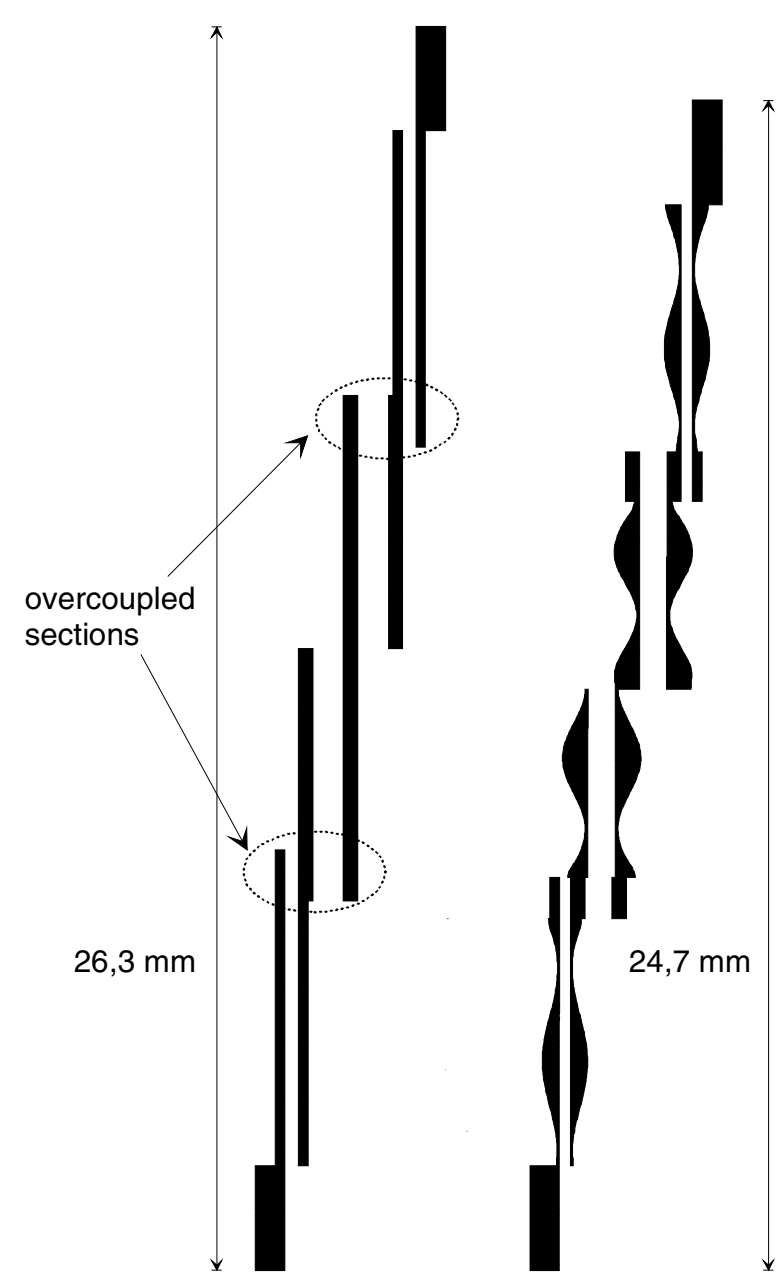

Fig. 4. Layouts of the overcoupled-resonator filter and of the dual approach filter.
It explains why the final filter length (fig. 4) is shorter than the initial one. The layouts in fig. 4 are given for an alumina substrate $\left(h=0.635 \mathrm{~mm}, \varepsilon_{r}=9.6, t=5 \mu \mathrm{m}\right.$, $\tan \delta=0.004)$.

The time of about 24s taken by simulation of 201 frequency points on a standard PC remains low compared to the duration of EM simulations and enables the use of optimization processes. Moreover, the layout generation is as simple as for built-in components. It is worth underlining that the substrate and metal losses and dispersion were all taken into account in circuit and EM simulations (Agilent Momentum). To avoid the use of too small strip widths and then limit the increase of metal losses, the optimization process was constrained by size limitations. Fig. 5 compares the $S_{21}$ simulated parameters given by the conventional filter to those of filters with overcoupled-resonator or mixed approach (overcoupling plus nonuniform). Simulations evidenced the rejection of the first three spurious passbands by more than $27 \mathrm{~dB}$.

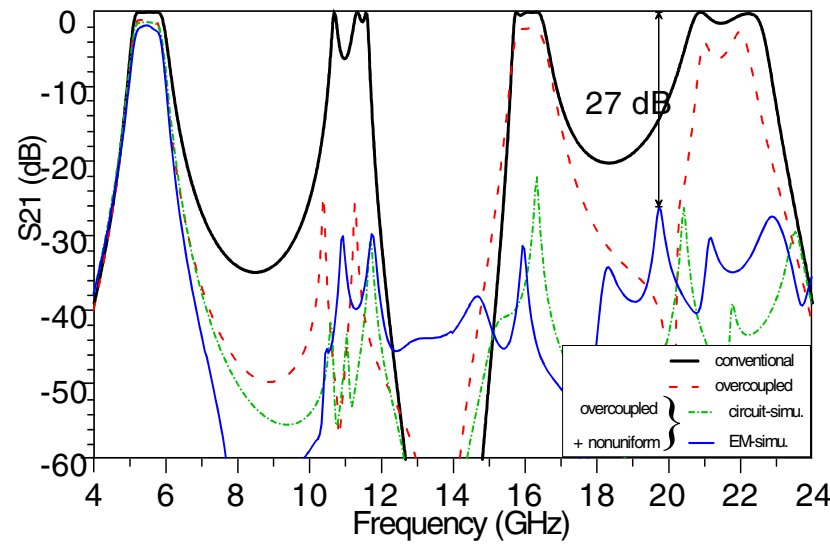

Fig. 5. $S_{21}$ parameters for the conventional, the overcoupled and the mixed approach filters.

Finally, Figure 6 shows the overall agreement between experiment results and electromagnetic response.

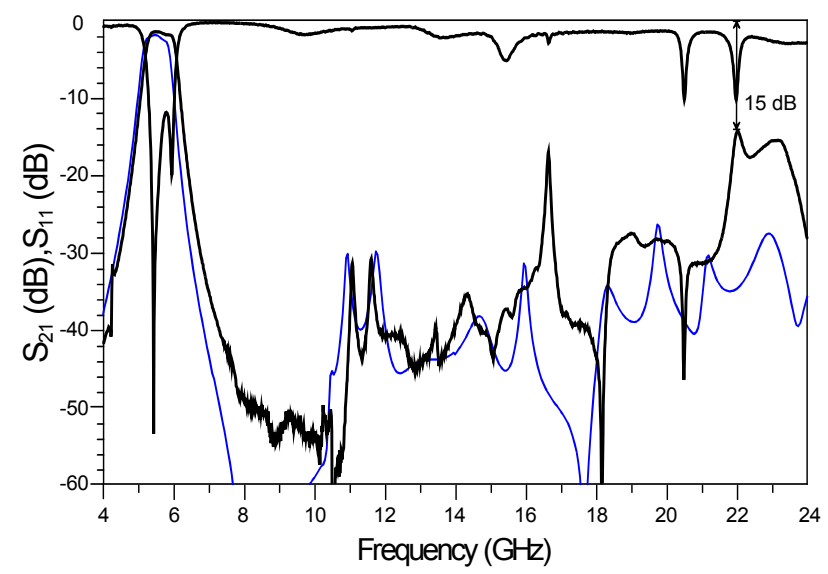

Fig. 6. Measured $S_{11}$ and $S_{21}$ parameters (thick line) and simulated $S_{2 I}$ parameter (blue thin line).

The third harmonic $\left(4 f_{c}\right)$ is only rejected by $15 \mathrm{~dB}$. The $3 \mathrm{~dB}$ bandwidth still shows a gently shift and is defined between 5.2 and $6 \mathrm{GHz}$. The open-end effect of the three-coupled microstrip line sections was modeled by 
open stubs; it may partly explain this residual shift. Insertion losses in the passband are about $1.5 \mathrm{~dB}$ with return losses below $-12 \mathrm{~dB}$.

\section{CONCLUSION}

We proposed several new models designed to facilitate the manipulation of arbitrary-shape single or coupled lines and allow accurate circuit simulations. The performances of a coupled-line microstrip filter were improved by introducing a new symmetrical perturbation via hierarchical components. Association of this technique to over-coupled resonators in a 3-order filter suppressed the first three spurious passbands. Such models can be used in several applications, e.g. harmonic suppression in hairpin filters [5], and will facilitate the design of wideband couplers [6]. Moreover, in order to minimize radiation losses at millimeter wavelength, impedance steps can be replaced through this approach with easily-introduced and -handled nonuniform tapers. Following [7] and using this new CPW hierarchical model will allow one to design matched tapers for better on-wafer measurements.

\section{REFERENCES}

[1] M. Le Roy, A. Pérennec, S. Toutain, and L. C. Calvez, "The Continuously Varying Transmission Lines Technique - Application to Filter Design," IEEE Transactions on Microwave Theory and Techniques, vol. 47, pp. 1680-1688, Sept. 1999.

[2] T. Lopetegi, A. G. Laso, F. Falcone, F. Martin, J. Bonache, J. Garcia, L. Pérez-Cuevas, and M. Sorolla, "Microstrip "Wiggly-Line" Bandpass Filters With Multispurious Rejection", IEEE Microwave and Wireless Component Letters, Vol. 14, pp. 531-533, Nov. 2004.
[3] A. Riddle, "High Performance Parallel Coupled Microstrip Filters", IEEE Microwave Theory and Techniques Symposium Digest, vol.1, pp. 427-430, 25-27 May 1988.

[4] S.W. Ting, K. P. Lei, C.P. Chiang, and K. W. Tam, "Novel Coupled Line Microstrip Bandpass Filter with $2^{\text {nd }} \& 3^{\text {rd }}$ Spurious Passbands Suppression", $32^{\text {nd }}$ European Microwave Conference, pp. 1141-1143, Milan 2002.

[5] S. I. Hong, S. W. Fok, W. W. Choi, K. W. Tam and R. P . Martins, "A Novel Wiggly-Line Hairpin Filter with $2^{\mathrm{Nd}}$ Spurious Passband Suppression", $34^{\text {th }}$ European Microwave Conference, pp. 1125-1128, Amsterdam, 2004.

[6] S. Uysal, "Nonuniform Line Microstrip Directional couplers and Filters", Norwood, Artech House, 1993.

[7] A.H. Hammade, A.B. Kouki, and F.M. Ghannouchi, "A CAD-Suitable Approach for the analysis of Nonuniform MMIC and MHMIC Transmission Lines, IEEE Transactions on Microwave Theory and Techniques, vol. 44, pp. 1614-1617, Sept. 1996. 